Bulletin of the Natural History Museum, 2019, 12: 179-207.

Received 09 Nov 2018; Accepted 12 Dec 2018.

doi:10.5937/bnhmb1912179P

UDC: $595.733(497.7)$

Original scientific paper

\title{
AQUATIC AND SEMIAQUATIC HETEROPTERA OF SERBIA
}

\author{
LJILJANA PROTIĆ ${ }^{1 *}$, BOJANA NADAŽDIN ${ }^{2}$ \\ ${ }^{1}$ Natural History Museum in Belgrade, 11000 Belgrade, Njegoševa 51, Serbia, \\ e-mail: 1jilja.protic@gmail.com \\ ${ }^{2}$ HabiProt, Janka Čmelika 28a 3/25, 21000 Novi Sad, Serbia
}

This paper includes a list of 62 species of Heteroptera (Nepomorpha and Gerromorpha), categorized into 12 families: Nepidae (2), Belostomatidae (1), Corixidae (25), Naucoridae (1), Aphelocheiridae (1), Notonectidae (6), Pleidae (1), Mesoveliidae (1), Hebridae (3), Hydrometridae (2), Velidae (9) and Gerridae (10). Two species are new for the fauna of Serbia: Hesperocorixa moesta (Fieber, 1848) and Velia (Plesiovelia) mancinii mancinii Tamanini, 1947.

Key words: Heteroptera, Nepomorpha, Gerromorpha, checklist, Serbia

\section{INTRODUCTION}

Heteroptera of aquatic and semiaquatic habitats classified into two infraorders, Nepomorpha (aquatic bugs) and Gerromorpha (semiaquatic bugs). The highest diversity of aquatic and semiaquatic bugs is in tropical areas. There are about 4800 species described on the global scale and over 600 species in the Palearctic (Reduciendo Klementova et al. 2015). They 
inhabit a wide range of habitats, from marine and shallow waters to arctic and high alpine. They are recorded on the water surface, on riverbanks in moss, aquatic and coastal vegetation. They are present in almost every freshwater biotope. Generally, they are predatory, while a smaller number of species is omnivorous.

Studies on aquatic and semiaquatic true bugs in Serbia has been neglected in comparison to terrestrial species. Individual records of aquatic and semiaquatic bugs are included in many faunistic papers (Divac 1907, Kormilev 1936, 1938, 1939, 1939a, 1943, Protić 1985, 1990, 1992, 1998, 2011a, 2011b, 2019, Protić et al. 2017, 2017a, Protić \& Živić 2007, Protić \& Živić 2012, 2013, Šeat 2011, 2013, 2017, Šeat \& Nadaždin 2015, 2018, Šeat et al. 2016, Živić et al. 2007, Živojinović 1950).

The first check-list of Heteroptera of Serbia (Protić 1993/1994) and the Catalogue of the Heteroptera fauna of Yugoslav countries (Protić 1998) included lists of aquatic and semiaquatic true bugs in Serbia. After these publications, numerous new data were collected new species were recorded for Serbia, while other species were recorded in new localities.

The aim of this paper is to publish for the first time all-including data overview on species from infraorders Nepomorpha and Gerromorpha, stored at the Entomological Collection of the Natural History Museum in Belgrade (NHMBEO) and Alciphron database, along with an overview of references for previously published records. Most of specimens were collected by aquatic sampling net (benthic sampling or sweeping of aquatic plants) and some specimens were sampled in light trap.

In the Entomological Collection of the Natural History Museum in Belgrade, aquatic and semiaquatic true bugs are categorized by families and genera, but until recently there were some unidentified stored specimens. In the last few years, specimens from the collection were determined with help of our colleagues Ping-Ping Chen and Nico Nieser (Naturalis Biodiversity Centre, Leiden, The Netherlands) and Gavril Marius Berchi (West University of Timişoara, Romania).

The online database "Alciphron" a database for collecting data on insects in Serbia, was established in 2012 and it is available on the Internet since 2014 (HabiProt 2014-2019). The administrators for Heteroptera are Jelena Šeat and Bojana Nadaždin. There are numerous participants in the database, and they entering field data on a daily basis (locations where specimens were found and date and photograph of each specimen), while administrators identify or verify each recording. At the time of writing this paper, the database includes 233 records of aquatic and semiaquatic true bugs identified to species level. Most of the recorded specimens are stored in private collections of the second author and Jelena Šeat, while a few are 
stored at the Faculty of Science at the University of Kragujevac. Certain specimens were identified only from photographs (such records are marked with " $F$ " in the species list).

During the processing of the material and preparation for writing this paper, it was very useful to compare the papers on aquatic and semiaquatic bugs from neighboring and other European countries. All of these data are important for understanding biology and ranges of certain species: Andersen 1982, Benedek 1970, Berchi 2013, Berchi \& Kment 2015, Berchi et al. 2016, Boda et al. 2015, Cianferoni 2013, 2019, Cianferoni \& Nardi 2013, Csabai et al. 2017, Damgaard 2006, Fent et al. 2011, Gogala 2003, Jansson 1986, Josifov 1986, 1999, Josifov \& Simov 2006, Kanyukova 2006, Kment \& Beran 2011, Rabitsch \& Zettel 2000, Schuster 1983, Soós et al. 2009, 2010, Tamanini 1959, Wróblewski 1980, Zimmermann 1982, Zimmermann \& Scholl 1993.

The order of families and species follows the Catalogue of the Heteroptera of the Palearctic Region (Aukema \& Rieger 1995; Aukema et al. 2013). Specimens were determined by using identification keys and individual taxonomic papers (Andersen 1996, Dahl 2005, Jaczewski 1962, Jaczewski \& Wróblewski 1976, 1978, Jansson 1986, 1996, Poisson 1957, Savage 1989, 1990, Stichel 1955-1956, Wachmann et al. 2006).

\section{RESULTS}

\section{List of aquatic and semiaquatic true bugs in Serbia}

\section{NEPOMORPHA}

Nepidae Laterille, 1802

1. Nepa cinerea Linnaeus, 1758

NHMBEO - Plavna: Zamna, r. 16.06.1987. 1ex. leg. Lj. Protić; Beograd: Bavanište 22.03.1990. 12ex. leg. M. Živković; Vlasinsko Jezero (lake): toward Crna Trava 28.07.1990. 3ex. leg. Lj. Protić; Avala: Čarapića Brest 01.06.1996. 2ex. leg. V. Žikić; Beljanica: Kanjon Suvaje 16.07.2004 1ex. leg. M. Vujanić; Mt. Jastrbac: Jezero (lake) 18.04.2007. 1ex., 07.05.2007. 1ex., 28.07.2007. 1ex., 16.09. 2007. 1ex., 17.09.2007. 1ex. leg. I. Parezanović.

Alciphron database - Stara Pazova 03.04.2011. leg. B. Hric (F); Kosovska Kamenica: Berivojce 15.04.2013. leg. B. Milošević (F); Beograd: Babe 24.05.2019. leg. M. Popović (F); Beograd: Grocka 26.04.2014. leg. U. Vezenković (F); Beograd: Kaluđerica 13.04.2017. leg. M. Vujić (F); Beograd: Beli Potok 12.10.2017., 20.05.2018. leg. D. Ćoso (F); Avala: Čarapićev Brest 06.01.2018. leg. D. Ćoso (F), 14.09. 
2019. leg. M. Vujić (F); Knić: Boračka Reka, r. 03.05.2018. leg. F. Vukajlović; Kragujevac: Grošnička Reka, r. 09.05.2018. leg. F. Vukajlović; Kragujevac: Šumaričko Jezero (lake) 11.05.2019. leg. F. Vukajlović; Bačka Palanka: Tikvara 16.04.2015. leg. I. Tot (F); Bačka Palanka: Karađorđevo 03.09.2015., 17.10.2018. leg. I. Tot (F); Grocka: Vrčin 23.04.2016., 25.03.2017., 07.01.2018., 30.03.2018. leg. M. Vujić (F); Donji Tavankut 28.04.2011. leg. G. Mesaroš; Palićko Jezero (lake) 17.04.2014. leg. G. Mesaroš; Kovačica 07.04.2017. leg. A. Husarik (F); Loznica: Cikote 16.08.2017. leg. M. Šćiban (F); Bor 21.08.2017. leg. M. Šćiban (F); Stara Planina: Pokrevenik 28.07.2015. leg. I. Tot (F); Pčinja: Trgovište 21.07.2018. leg. B. Nadaždin; Vranje: Moštanica 17.09.2018. leg M. Đurić (F); Vlasina: Polom 06.09.2019. leg. B. Nadaždin; Vlasina: Gadžini 03.09.2019. leg. M. Vujić; Vlasina: Veliki Čemernik 15.08.2019. leg. I. Tot.

Ref: Divac 1907, Kormilev 1936, Csiki 1940, Protić 1985, 1992, 1998, 2008, Protić \& Živić 2007, Šeat 2011, 2013, 2017, Protić \& Živić 2012, 2013, Protić et al. 2017, 2017a.

\section{Ranatra linearis (Linnaeus, 1758) (Fig. 1)}

NHMBEO - Beograd: Topčider 07.1955. 1 ex. leg. B. Salamon; Beograd: Ada Ciganlija 27.05.2000. 1ex. leg. S. Polak; Mt. Jastrebac: Jezero (lake) 07.05.2007. 1ex leg. I. Parezanović; Kovin: Malo Bavanište 24.06.2007. 1ex., 08.07.2007. 1ex. leg. M. Obradović.

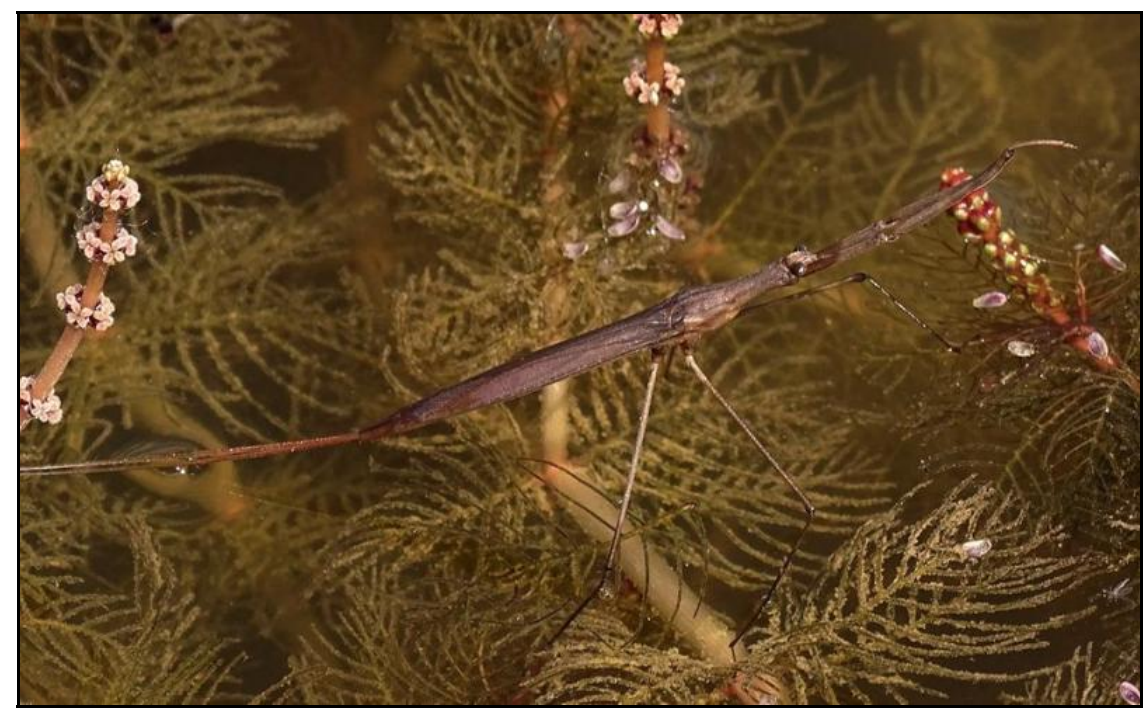

Fig. 1 - Ranatra linearis (photo Zoran Gavrilović).

Alciphron database - Bačka Palanka: Čarda "Florida" 10.10.2010. leg. I. Tot; Bačka Palanka: Tikvara 06.10.2016., 25.10.2016., 02.07.2018., 
17.07.2019. leg. I. Tot; Nadalj 18.06.2014. leg. R. Mirić (F); Kragujevac: Grošničko Jezero (lake) 25.03.2017. leg. F. Vukajlović; Vršac: Gradsko Jezero (lake) 16.05.2017. leg. Z. Gavrilović (F); Stari Begej: Traktor Bara 06.09.2018. leg. B. Nadaždin.

Ref.: Horváth 1903, Divac 1907, Protić 1998, 2008.

Belostomatidae Leach, 1815

3. Lethocerus patruelis (Stål, 1854)

Alciphron database - Raška: Supnje 10.06.2015. leg. F. Vukajlović.

Ref.: Kormilev 1936.

Corixidae Leach, 1815

MiCRONECTINAE Jaczewski, 1924

4. Micronecta (Dichaetonecta) pusilla (Horváth, 1895)

Ref.: Horváth 1897.

5. Micronecta (Dichaetonecta) scholtzi (Fieber, 1860)

Ref.: Divac 1907, Kormilev 1936.

6. Micronecta (Micronecta) carpatica Wróbelowski, 1958

Ref.: Kment 2006.

7. Micronecta (Micronecta) minutissima (Linnaeus, 1758)

NHMBEO - Kovin: Malo Bavanište 08.07.2007. 1ex. leg. M. Obradović.

Ref.: Protić 2011b.

8. Micronecta (Micronecta) poweri poweri (Douglas \& Scott, 1869)

Ref.: Protić 1998, 2011 a.

CYMATIAINAE Walton, 1940

9. Cymatia coleoptrata (Fabricius, 1777)

Ref.: Horváth 1897; Kormilev 1936, 1938; Protić 1985, 1998; Protić et al. $2017 \mathrm{a}$.

10. Cymatia rogenhoferi (Fieber, 1864)

Alciphron database: Sombor 22.07.2016. leg. D. Vajgand.

Ref.: Horváth 1897, Kormilev 1936.

CORIXINAE Leach, 1815.

11. Arctocorisa germari (Fieber, 1848)

Ref.: Protić 2011a.

12. Callicorixa praeusta praeusta (Fieber, 1848)

NHMBEO - Beograd: Veliko Selo 08.07.2006. leg. A. Stojanović. 
Ref.: Protić \& Živić 2013, Šeat 2013.

New for Catalogue of the Heteroptera of the Palaearctic Region.

13. Corixa affinis Leach, 1817

NHMBEO - Negotin: Mihajlovac, Djala Mare 19/20.07.2017. 1ex. legs. A. Stojanović \& M. Jovanović. Attracted by light.

Ref.: Kormilev 1936; Protić 1985, 2011 a; Protić et al. 2017 a.

14. Corixa panzeri Fieber, 1848

Ref.: Protić 2011a.

15. Corixa punctata (Illiger, 1807)

NHMBEO - Beograd: Banjica 22.03.1999. 2ex. leg. M. Živković.

Ref.: Horváth 1897, Langhoffer 1899, Živojinović 1950, Protić 1998, 2011a, Protić \& Živić 2012.

16. Hespercorixa linnaei (Fieber, 1848)

NHMBEO - Kovin: Malo Bavanište 08.07.2007. 1ex. leg. M. Obradović.

Alciphron database - Riđica 13.08.2015. leg. G. Mesaroš; Sombor 23.06.2016., 22.07.2016., 26.07.2016., 27.07.2016., 06.08.2016. leg. D. Vajgand; Čelarevo 20.09.2015., 30.07.2016. leg. D. Vajgand.

Ref.: Protić 2011b, Protić \& Živić 2013, Protić et al. 2017a.

17. Hesperocorixa moesta (Fieber, 1848)

New species of the fauna of Serbia.

Alciphron database - Vlasina: Dejanova Reka, r. 26.05.2019. leg. M. Vujić.

New for Catalogue of the Heteroptera of the Palaearctic Region.

18. Hesperocorixa parallela (Fieber, 1860)

Ref.: Protić \& Živić 2013.

New for Catalogue of the Heteroptera of the Palaearctic Region.

19. Hespercorixa sahlbergi (Fieber, 1848)

Alciphron database - Pešter: Braćak 13.10.2018. leg. B. Nadaždin; Pešter: Karajukića Bunari 14.10.2018. leg. B. Nadaždin.

Ref.: Kormilev 1936, Protić 2011a.

20. Paracorixa concinna concinna (Fieber, 1848)

NHMBEO - Deliblatski Pesak: Devojački Bunar 24.04.2006. 1ex. leg. A. Stojanović.

Alciphron database - Sombor 20.05.2015., 22.07.2016., 27.07.2016, 06.08.2016. leg. D. Vajgand; Gakovo 05.06.2015. leg. J. Šeat. 
Ref.: Horváth 1897, Protić 2011a.

\section{Sigara (Pseudovermicorixa) nigrolineata nigrolineata (Fieber,1848)}

NHMBEO: Avala 13.07.1941. 2ex. leg. B. Petrov; Bavanište 22.03. 1990. 4ex. leg. M. Živković; Mala Moštanica: Žuto Brdo 13.05.2006. 4ex., 14.04.2007. 1ex. leg. A. Stojanović; Stari Slankamen: Črnok 23/24.08.2019. 7ex. leg. A. Stojanović. Attracted by light.

Alciphron database - Pešter: Braćak 13.10.2018. leg. B. Nadaždin; Pešter: Karajukića Bunari 14.10.2018. leg. B. Nadaždin.

Ref.: Protić 2011a, Protić \& Živić 2012, 2013, Šeat 2011.

22. Sigara (Retrocorixa) limitata limitata (Fieber, 1848)

Ref.: Horváth 1897; Kormilev 1936; Protić 1990, 1998, 2011a; Protić, Živić 2012; Šeta 2017.

23. Sigara (Retrocorixa) semistriata (Fieber, 1848)

NHMBEO - Beograd: Veliko Selo 08.07.2006. 5ex. leg. A. Stojanović.

Ref.: Josifov 1986, Protić 2011b.

New for Catalogue of the Heteroptera of the Palaearctic Region.

24. Sigara (Sigara) striata (Linnaeus, 1758)

NHMBEO - Beograd: Veliko Selo 08.07.2006. 1ex. leg. A. Stojanović; Kovin: Malo Bavanište 21.04.2007. 1 ex., 24.06.2007. 3ex., 30.06. 2007. 3ex., 08.07. 2007. 15ex., 18.07.2007. 3ex., 16.09.2007. 9ex., 30. 06.2007.m 1ex., 15.07.2007. 18ex., leg. M. Obradović; Boljevci: Crni Lug 16.09.2016. 2ex. leg. A. Stojanović; Pančevo: Veliko Blato, Tamiš, r. 25/26.05.2019. 5 ex. leg. A. Stojanović; Stari Slankamen, Dunav, r. 18/19.06.2018. 1ex. leg. A. Stojanović; Beograd: Zuce, Malozučke Livade, meadows, 28/29.06.2019. 13ex. leg. A. Stojanović; Stari Slankamen: Čarnok 23/24.08.2019. 6ex. leg. A. Stojanović. Attracted by light.

Alciphron database - Stari Slankamen 09.06.2012. leg. B. Hric (F); Stara Pazova 22.07. 2012. leg B. Hric (F); Riđica 13.08.2015. leg. G. Mesaroš; Gakovo 09.06.2013., 05.06.2015. leg. J. Šeat; Beograd: Beli Potok 24.05.2018. leg. D. Ćoso (F); Sombor 20.05.2015., 23.06.2016., 22.07.2016., 26.07.2016., 27.07.2016., 01.08.2016., 02.08.2016., 06.08. 2016. leg. D. Vajgand; Čelarevo 12.09.2015., 16.09.2015., 17.09.2015., 19.09.2015., 20.09.2015. leg. D. Vajgand; Fruška Gora: Stražilovo 29.06.2019. leg. B. Nadaždin; Pešter: Karajukića Bunari 14.10.2018. leg. B. Nadaždin.

Ref.: Kormilev 1936, Živojinović 1950, Protić 2011a, Protić \& Živić 2012. 
25. Sigara (Subsigara) distincta (Fieber, 1848)

Ref.: Kormilev 1936, Protić 1985, 2011 a.

26. Sigara (Subsigara) falleni (Fieber, 1848)

NHMBEO - Stara planina: Kamenička Reka, r. 05.08.2001. 2ex. leg. I. Živić; Beljanica; Boljevci: Crni lug 16.09.2006. 2ex. leg. A. Stojanović; Kovin: Malo Bavanište 10.06.2007. leg. M. Obradović; Stari Slankamen: Čarnok 23/24.08.2019. 3ex. leg. A. Stojanović; Beograd: Zuce, Malozučke Livade (meadows) 28/29.06.2019. 3ex. leg. A. Stojanović. Attracted by light.

Alciphron database - Sombor 23.06.2016., 22.07.2016., 26.07.2016., 27.07.2016., 01.08.2016., 06.08.2016. leg. D. Vajgand; Čelarevo 20.09.2015. leg. D. Vajgand; Gakovo 05.06.2015. leg. J. Šeat; Bačka Palanka: Tikvara 10.06.2015. leg. I. Tot.

Ref.: Langhoffer 1899, Kormilev 1936, Protić 1985, 2011a, Protić \& Živić 2007, Protić et al. 2017a.

27. Sigara (Subsigara) iactans Jansson, 1983

Ref.: Protić \& Živić 2012.

New for Catalogue of the Heteroptera of the Palaearctic Region.

28. Sigara (Vermicorixa) lateralis (Leach, 1818)

NHMBEO - Bavanište 22.03.1990. 8ex. leg. M. Živković; Mala Moštanica: Žuto Brdo 14.04.2007. 1ex. leg. A. Stojanović; Pančevo: Veliko Blato, Tamiš, r. 25/26.05.2019. 6 ex. leg. A. Stojanović; Stari Slankamen 18/19.05.2018. 1ex. leg. A. Stojanović; Topola: Banja 17/18.08.2018. 2 ex. leg. A. Stojanović; Beograd: Zuce, Malozučke Livade, meadows 28/29.06.2019. 6 ex. leg. A. Stojanović. Attracted by light.

Alciphron database - Riđica 13.08.2015. leg. G. Mesaroš; Sombor 20.05.2015., 23.06.2016., 22.07.2016., 27.07.2016., 31.07.2016., 01.08. 2016., 06.08.2016. leg. D. Vajgand; Sombor: Šikara 04.10.2013. leg. J. Šeat; Čelarevo 12.09.2015., 19.09.2015., 20.09.2015., leg. D. Vajgand; Gakovo 05.06.2015., 23.06.2015. leg. J. Šeat; Fruška Gora: Stražilovo 29.06.2019. leg. B. Nadaždin; Pešter: Braćak 13.10.2018. leg. B. Nadaždin.

Ref.: Divac 1907, Kormilev 1936, Živojinović 1950, Protić 1998, 2011a, Protić \& Živić 2012, 2013, Šeat, Nadaždin 2015, Protić et al. $2017 \mathrm{a}$. 
Naucoridae Leach, 1815

29. Ilyocoris cimicoides cimicoides (Linnaeus, 1758)

NHMBEO: Topčider 07.1956. 1ex. leg. B. Salamon; Beograd: Ilićev Dunavac 17.06.1994. 1ex. leg. V. Žikić; Beograd: Ada Ciganlija 05.2006. 2ex. leg. I. Parezanović; Kovin: Malo Bavanište 15.04.2007. 9ex., 21.04.2007. 5ex., 08.07.2007. 4ex., 15.07.2007. 2ex., 30.08.2007. 1ex., 16.09.2007. 15ex., 14.10.2007. 3ex. leg. M. Obradović; Novi Sad 04.11.2009. 1ex. leg. D. Savić.

Alciphron database - Stara Pazova 17.04.2010., 05.03.2012. leg. B. Hric (F); Bačko Gradište 08.03.2013. leg. M. Šćiban (F); Bačka Palanka: Tikvara 11.03.2014., 03.09.2015. leg. I. Tot; Bački Vinogradi 18.07.2014. leg. J. Šeat; Palićko Jezero (lake) 17.04.2014. leg. G. Mesaroš; Beograd: Veliko Ratno Ostrvo (island) 24.04.2017. leg. M. Vujić (F).

Ref.: Horváth 1903; Divac 1907; Kormilev 1936; Protić 1985, 1998, 2008; Protić et al. 2017a.

Aphelocheiridae Fieber, 1851

30. Aphelocheirus aestivalis (Fabricius, 1794)

Ref.: Živić et al. 2007, Protić \& Živić 2007, Protić \& Živić 2012.

Notonectidae Latreille, 1802

31. Anisops sardeus Herrich-Schaeffer, 1849

Alciphron database - Palićko Jezero (lake) 03.08.2019. leg. S. Čokić.

Ref.: Šeat et al. 2019.

New for Catalogue of the Heteroptera of the Palaearctic Region.

32. Notonecta glauca glauca Linnaeus, 1758 (Fig. 2)

NHMBEO: Beograd: Topčider 01.06.1942. 1ex., 22.07.1942. 1ex., 04.08.1942. 1 ex. leg. B. Petrov; Mt. Jastrebac 18.07.1965. 1ex. leg. D. Čubrilović; Rajac 13.07.1979. 3ex. leg. Lj. Kašić; Vlasinsko Jezero (lake): toward Crna Trava 28.07.1990. 4ex. leg. Lj. Protić; Fruška Gora: Vrdnik 04.06.2000. 1 ex. leg. M. Mišković, Beograd: Resnik, Kružni Put 06.07.2002. 1ex. leg. A. Stojanović; Valjevo: Donji Taor 06.08.2003. 8ex. leg. M. Vujanić, Beljanica: Lisine 19.07.2004. 1ex. leg. M. Vujanić; Beljanička Reka, r. 21.07.2004. 1ex. leg. M. Vujanić; Boljevci: Crni Lug 16.09.2006 1ex. leg. A. Stojanović; Mt. Jastrebac: Jezero (lake) 18.04.2007. 1ex., 07.05.2007.1ex., 08.07.2007. 3ex., 17.07.2007. 2ex., 28.07.2007. 3ex., 23.09.2007. 2ex., 14.10.2007. 2ex. leg. I. Parezanović; Kovin: Malo Bavanište 10.06.2007. 1ex., 24.06. 2007. 1ex., 08.07.2007. 1ex. leg. M. Obradović; Beograd: Veloko Selo 
21.04.2007. 1ex. leg. A. Stojanović; Lazarevac: Stubica 19.07.2008. 2ex. leg. A. Stojanović; Mala Moštanica: Žuto Brdo 10.07.2010. 2ex. leg. A. Stojanović.

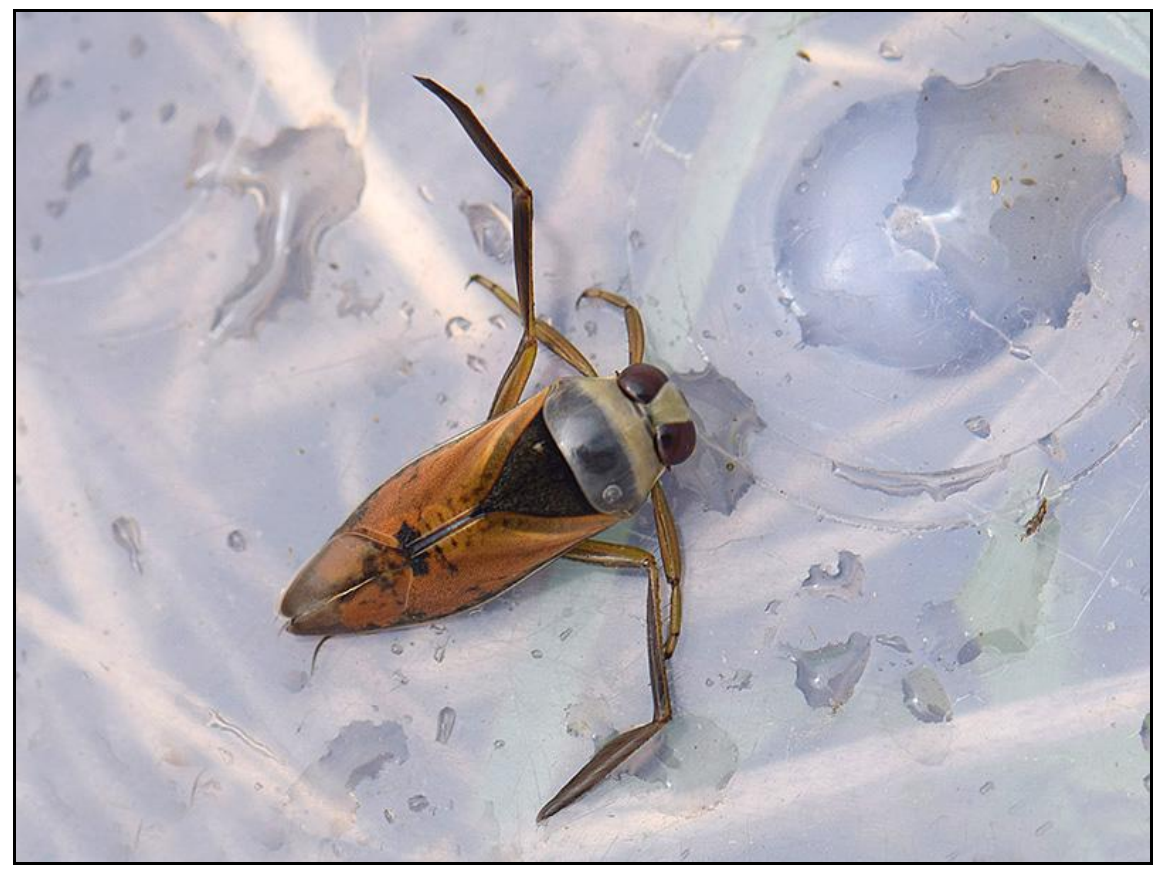

Fig. 2 - Notonecta glauca (photo Ivan Pančić).

Alciphron database - Cer: Planinarski Dom "Lipove vode" 06.07.2012. leg. I. Radulović, 29.09.2017. leg. A. Đukić; Kosovska Kamenica: Berivojce 25.10.2013., 02.11.2013. leg. B. Milošević (F); Kupusina 20.06.2014. leg. I. Kovač (F); Zrenjanin 22.04.2015., 07.06.2015., 17.06.2018. leg. I. Pančić (F); Bačka Palanka: Tikvara 03.09.2015. leg. I. Tot; Stara Pazova 31.03.2016. leg. B. Hric (F); Stara Planina: Temska 21.07.2014. leg. M. Šćiban; Novi Sad: Kamenički Park 06.02.2018. leg. N. Milić (F); Pčinja: Šaprance 22.07.2018. leg. B. Nadaždin; Pešter: Karajukića Bunari 14.10.2018. leg. B. Nadaždin; Vlasina: Gadžini 03.09.2019. leg. M. Vujić.

Ref.: Protić 2008, Protić \& Živić 2007, Protić \& Živić 2012, 2013, Protić et al. 2017, Protić et al. 2017a, Šeat 2011, 2017.

33. Notonecta lutea Müller, 1776

Ref.: Kormilev 1938, Protić 1998.

34. Notonecta maculata Fabricius, 1794

Ref.: Protić 1985, Protić \& Živić 2013, Šeat, Nadaždin 2018.

New for Catalogue of the Heteroptera of the Palaearctic Region. 
35. Notonecta obliqua Thunberg, 1787

NHMBEO: Mt. Jastrebac: Jezero (lake) 08.07.2007. 1ex., 14.10.2007. lex. leg. I. Parezanović.

Ref.: Živojinović 1950.

36. Notonecta viridis Delcourt, 1909

NHMBEO: Beograd: Topčider 22.07.1942. 2 ex. leg. B. Petrov; Beograd: Topčider 07.1959. 1ex. leg. B. Salamon; Barič 09.06.2008. 1ex. leg. S. Rauš; Miroč 19.07.2005. 3ex., 20.07.2005. 1ex. leg. M. Vujanić; Ljig: Kadina Luka 15.06.2013. 1ex leg. A. Stojanović; Beljanica: Lisine 16.07.2004. 9ex., 19.07.2004. 2ex. leg. M. Vujanić; Mt. Jastrebac: Jezero (lake) 08.07.2007. 2ex., 28.07.2007. 1ex., 17.09.2007. 2ex., 23.09.2007. 5ex., 14.10.2007. 1ex. leg. I. Parezanović; Beograd: Veliko Selo 21.04.2007. 1ex leg. A. Stojanović.

Ref.: Živojinović 1950; Protić 1990, 1998; Protić \& Živić 2012.

New for Catalogue of the Heteroptera of the Palaearctic Region.

Pleidae Fieber, 1851

37. Plea minutissima minutissima Leach, 1817

NHMBEO: Zemun 25.08.1941 1ex. leg. B. Kodrič; Boljevci: Crni Lug 16.09.2006. 6ex. leg. A. Stojanović; Kovin: Malo Bavanište 08.07. 2007. 5ex. leg. M. Obradović.

Alciphron database - Zrenjanin: Lukino Selo 30.06.2013. leg. I. Pančić (F); Sombor: Šikara 04.10.2013. leg. J. Šeat; Bački Vinogradi 18.07. 2014. leg. J. Šeat; Pešter: Karajukića Bunari 14.10.2018. leg. B. Nadaždin.

Ref.: Horváth 1903, 1918, Divac 1907, Kormilev 1936, Csiki 1940, Protić 1998, Protić et al. 2017a.

\section{GERROMORPHA}

Mesoveliide Douglas \& Scott, 1867

38. Mesovelia furcata Mulsant \& Rey, 1852

NHMBEO - Pančevački Rit: Kovilovo 02.09.2006. 1 ex. leg. A. Stojanović; Kovin: Malo Bavanište 08.07.2007. 2ex., 14.10.2007. 2ex. leg. M. Obradović.

Alciphron database - Zrenjanin 27.06.2013. leg. I. Pančić (F).

Ref.: Protić 2011b, Protić \& Živić 2012, Protić et al. 2017a.

Hebridae Amyot \& Serville, 1843

39. Hebrus (Hebrus) montanus Kolenati, 1857

Ref.: Kment, Jindra 2005. 
40. Hebrus (Hebrus) pusillus pusillus (Fallén, 1807)

NHMBEO: Plavna: Reka Zamna, r. 16.06.1987. 1ex. leg. Lj. Protić; Beograd: Topčiderski Park 24.06.2006. 5ex. leg. A. Stojanović.

Alciphron database - Bački Vinogradi 24.09.2012. leg. G. Mesaroš; Novi Sad 02.04.2015. leg. M. Śćiban.

Ref.: Protić et al. 2017a.

41. Hebrus (Hebrusella) ruficeps Thomson, 1871

Alciphron database - Bačko Gradište 20.03.2013., 08.04.2013., 11.03. 2018. leg. M. Šćiban.

Ref.: Horváth 1897, Protić 1998, Protić et al. 2017a.

Hydrometridae Billberg, 1820

42. Hydrometra gracilenta Horváth, 1899

NHMBEO: Kovin: Malo Bavanište 15.04.2007. 1ex.; 08.07.2007. 1ex. leg. M. Obradović;

Ref.: Šeat et al. 2016.

New for Catalogue of the Heteroptera of the Palaearctic Region.

43. Hydrometra stagnorum (Linnaeus, 1758)

NHMBEO - Plavna r. Zamna 16.06.1987. 3 ex. leg. Lj. Protić; Smederevo 18.06.1988. 2ex. leg. G. Mesaroš; Đerdap: Mosna 19.07. 2005. 11ex. leg. Lj. Protić; Mala Moštanica: Žuto Brdo 22.06.2002. 4ex. leg. A. Stojanović; Beograd: Resnik 06.07.2002. 1ex. leg. A. Stojanović; Mt. Jastrebac: Jezero (lake) 18.04.2007. 2ex., 07.05.2007. 3ex., 13.05.2007. 1ex., 17.09.2007. 2ex.; Mosna: Porečka Reka, r. 19.07.2005. 7ex. leg. Lj. Protić; Lazarevac: Stubica, 12.06.2010. 3ex. leg. A. Stojanović.

Alciphron database - Sokobanja: Vodopad Ripaljka (fall) 17.06.2015. leg. M. Popović (F); Novi Sad: Ledinci 06.04.2018. leg. M. Janković (F); Fruška Gora: Paragovo 16.04.2016. leg. M. Vujić (F); Grocka: Vrčin 09.10.2015. leg. M. Vujić (F); Loznica: Cikote 16.08.2017. leg. M. Šćiban (F); Stara Planina: Oreovica 15.07.2013. leg. M. Šćiban; Reljan 23.04.2019. leg. B. Nadaždin.

Ref.: Horváth 1903, 1918; Divac 1907; Schumacher 1918; Kormilev 1936; Protić 1985, 1992, 1998, 2008; Protić \& Živić 2007; Šeat 2011, 2013; Šeat, Nadaždin 2015, 2018; Protić et al. 2017, 2017 a.

Comment: Smederevo - unpublished record from the collection of Moravian Museum, Brno [correspondence, Petr Kment]. 
Veliidae Brullé, 1836

MicroveliINAE China \& Usinger, 1949

44. Microvelia (Microvelia) pygmaea (Dufour, 1833)

Ref.: Horváth 1903, Protić 1985, 1998.

New for Catalogue of the Heteroptera of the Palaearctic Region.

45. Microvelia (Microvelia) reticulata (Burmeister, 1835)

NHMBEO: Pančevački Rit: Kovilovo 02.09.2006. 1ex. leg. A. Stojanović.

Alciphron database - Zrenjanin 16.06.2013., 27.06.2013., 22.03.2015., 23.03.2015. leg. I. Pančić (F); Sombor: Šikara 04.10.2013. leg. J. Šeat.

Ref.: Horváth 1897, Protić 1990, 1998, Protić et al. 2017a.

46. Velia (Plesiovelia) affinis filippii Tamanini, 1947

NHMBEO: Mt. Jastrebac Jezero (lake) 08.07.2007. 1ex. leg. I. Parezanović; Topola: Banja, Zmajevac 25/26.07.2014. 1ex. leg. A. Stojanović. Attracted by light.

Ref.: Protić \& Živić 2007, Šeat 2013.

47. Velia (Plesiovelia) caprai caprai Tamanini, 1947

Ref.: Protić \& Živić 2007, Protić \& Živić 2012.

48. Velia (Plesiovelia) currens (Fabricius, 1794)

Ref.: Horváth 1897, Schumacher 1918, Kormilev 1936, Csiki 1940, Protić 1990, Šeat 2011.

49. Velia (Plesiovelia) mancinii mancinii Tamanini, 1947

New species in the fauna of Serbia.

Prokuplje 30.06.1941. leg. B. Petrov; Beograd: Avala 25.06.1941. leg. B. Petrov (Protić 1990, 2011a).

Comment: Velia (Velia) rivulorum (Fabricius) was misidentified in published records (Protić 1998, 2011a), they turned out to be Velia (Plesiovelia) mancinii mancinii Tamanini.

New for Catalogue of the Heteroptera of the Palaearctic Region.

50. Velia (Plesiovelia) sauli Tamanini, 1947

NHMBEO: Golubac 06.07.1956. 1ex. leg. J. Stančić; Beograd: Topčiderski Park 22.05.2004. 1ex. leg. A. Stojanović; Mala Moštanica: Žuto Brdo 22.06.2002. 1ex. 23.04.2011. 1ex. leg. A. Stojanović; Grocka: Vrčin 07.04.2007. 3ex. leg. A. Stojanović; Topola, s. Banja: Zmajevac 17.07.2015. 1ex. leg. A. Stojanović. 
Comment: Specimens in collection by Nikole Kormilev (Protić 1990) collected at localities Prokuplje and Avala are not V. rivulorum but $V$. sauli.

New for Catalogue of the Heteroptera of the Palaearctic Region.

51. Velia (Plesiovelia) serbica Tamanini, 1951

NHMBEO: Valjevo: Debelo Brdo 13.04.2002. 2ex, 14.04.2002. 1ex. leg. M. Vujanić; Rudnik: Gradovi 10.06.2003. 1ex. leg. Lj. Protić; Mt. Jastrebac: Jezero (lake) 08.07.2007. 1ex. leg. I. Parezanović; Goč 21.07.2011. 2ex. legs. M. Jovanović \& A. Stojanović.

Alciphron database - Grocka: Vrčin 19.11.2017. leg. M. Vujić (F); Stara planina: Oreovica 15.07.2013. leg. M. Šćiban; Pčinja: Isposnica Prohora Pčinjskog 24.04.2019. leg. B. Nadaždin; Vlasina: Dejanova Reka, r. 26.05.2019., 02.09.2019. leg. M. Vujić, 24.07.2019. leg. B. Nadaždin; Vlasina: Polom 23.07.2019., 06.09.2019. leg. B. Nadaždin; Vlasina: Gadžini 03.09.2019. leg. M. Vujić.

Ref.: Šeat 2013, 2017, Šeat, Nadaždin 2015, 2018.

New for Catalogue of the Heteroptera of the Palaearctic Region.

52. Velia (Velia) rivulorum (Fabricius, 1775)!

Ref.: Horváth 1903; Kormilev 1936; Csiki 1940; Protić 1985, 1992, 1998.

\section{Gerridae Leach, 1815}

\section{Aquarius najas (De Geer, 1773}

NHMBEO: Mt. Jastrebac: Jezero (lake) 21.08.2007. 7 ex., 23.09.2007. 6 ex., 14.10.2007. 1 ex. leg. I. Parezanović.

Alciphron database - Brestovačka Banja 29.04.2013. leg. P. Petrović (F); Kosovska Kamenica: Berivojce 17.07.2011. leg. B. Milošević (F); Sokobanja 17.06.2015. leg. M. Popović (F); Stara Planina: Temska 27.07.2015. leg. M. Milković (F); Pirot: Dag Banjica 30.08.2015. leg. I. Medenica (F); Valjevo: Belić 04.05.2013. leg. G. Mesaroš; Loznica: Cikote 16.08.2017. leg. M. Šćiban (F); Bor 04.07.2017. leg. M. Šćiban (F); Brusnica 03.04.2018. leg. M. Miljević (F); Arilje: Roge 20.05. 2018. leg. B. Nadaždin; Pčinja: Manastir Prohor Pčinjski 20.07.2018. leg. B. Nadaždin; Pčinja: Trgovište 21.07.2018. leg. B. Nadaždin; Pčinja: Dejance 15.09.2018. leg. B. Nadaždin; Vlasina: Dejanova Reka, r. 26.05.2019., 02.09.2019. leg. M. Vujić.

Ref.: Protić 1992, 1998, 2008, Protić \& Živić 2007, Šeat 2013, Šeat, Nadaždin 2015. 


\section{Aquarius paludum paludum (Fabricius, 1794)}

NHMBEO: Obedska Bara 1ex. 05.1954. leg. D. Čubrilović; Zlatar 6ex. 28.07.1979. Lj. Tričković Mt. Jastrebac: Jezero (lake) 18.04.2007. 4ex., 17.09.2007. 2ex. leg. I. Parezanović; Kovin: Malo Bavanište 23.07. 2006. 1ex. leg. M. Obradović.

Alciphron database - Zrenjanin 14.08.2013. leg. I. Pančić (F); 05.08. 2015. leg. M. Mareš (F); Kučaj: Dubašnica 03.05.2014. leg. P. Petković (F); Novi Beograd 23.06.2012. leg. M. Mareš (F); Žabalj 15.08.2015. leg. B. Budiša (F); Sopot 01.04.2016. leg. M. Đurić (F); Petničko Jezero (lake) 02.08.2014. leg. M. Jovanović (F); Vršac: Potporanj 09.06.2017. leg. Z. Gavrilović (F); Beograd: Ada Ciganlija 29.06.2019. leg. M. Mareš (F); Deliblato 13.04.2019. leg. I. Pančić; Carska Bara 06.09.2018. leg. B. Nadaždin.

Ref.: Horváth 1897, 1903, Kormilev 1936, Protić 1998, 2008, Protić \& Živić 2007, Šeat, Nadaždin 2015, 2018, Protić et al. 2017a.

55. Gerris (Gerris) argentatus Schummel, 1832 (Fig. 3)

NHMBEO: Deliblatski Pesak: Devojački Bunar 24.04.2006. 1 ex. leg. A. Stojanović; Kovin: Malo Bavanište 15.04.2007. 3ex., 21.04.2007. 2ex., 10.06.2007. 7ex., 24.6.2007. 8ex., 30.06.2007. 21ex., 08.07.2007. 9ex., 15.07.2007. 5ex., 23.07.2006. 3ex., 14.10.2007. 4ex. leg. Maja Obradović.

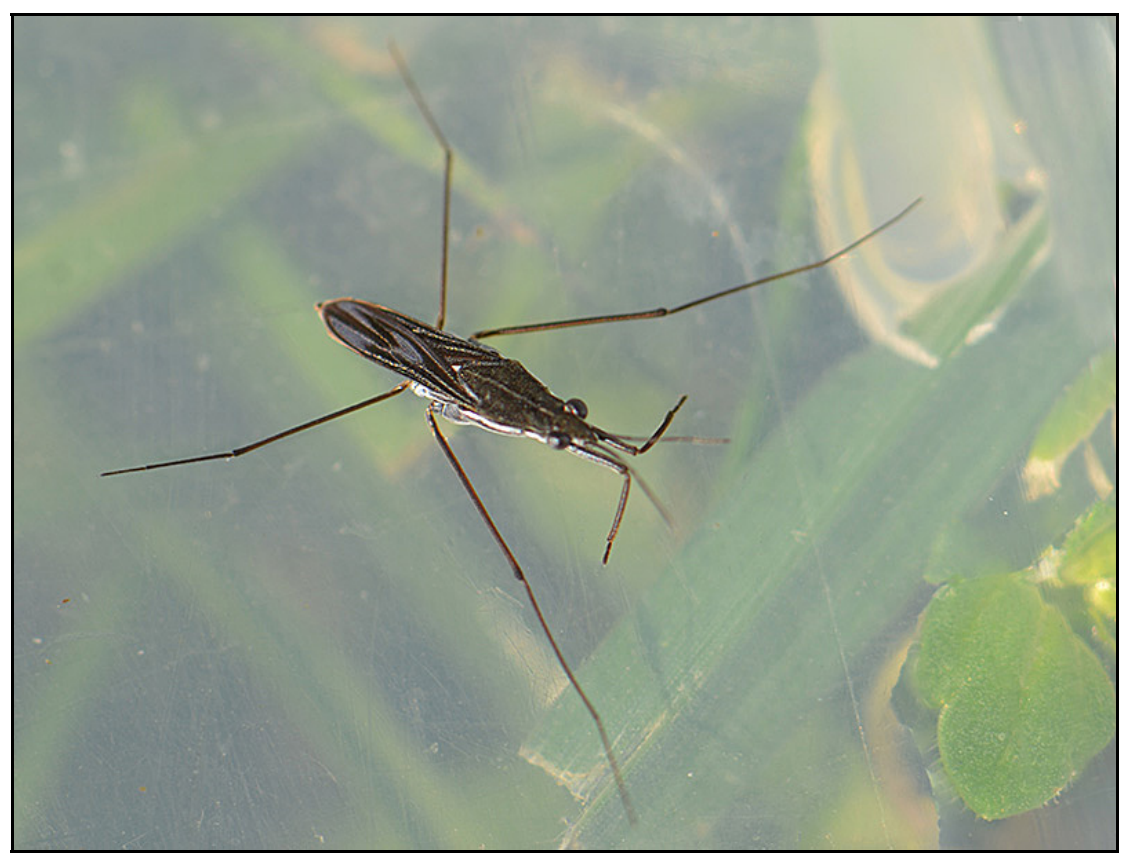

Fig. 3 - Gerris argentatus (photo Ivan Pančić). 
Alciphron database - Zrenjanin 22.04.2015. leg. I. Pančić (F); Bački Vinogradi 24.09.2012. leg. G. Mesaroš; Zasavica 27.10.2018. leg. M. Šćiban.

Ref.: Horváth 1897, Kormilev 1936, Protić 1990, 1998, Šeat, Nadaždin 2018.

56. Gerris (Gerris) costae fieberi Stichel, 1938

NHMBEO: Mt. Jastrebac: Jezero (lake) 07.05.2007. 2ex., 08.07.2007. 1ex.; Mala Moštanica: Žuto Brdo 13.05.2006. 3ex. leg. A. Stojanović; Valjevo: Donji Taor 08.08.2003. 3ex. leg. M. Vujanić.

Alciphron database - Obrež 18.03.2012. leg. B. Hric (F); Povlen: Makovište 15.05.2005. leg. M. Jovanović (F); Brusnica 11.04.2015. leg. M. Miljević (F); Sopotnica 13.07.2015. leg. Đ. Petrović, 15.07. 2015. leg. J. Šeat, 31.05.2016., 02.06.2016. leg. B. Nadaždin, 18.07. 2016. leg. I. Parenta; Stara Planina: Rudinje 19.07.2013. leg. M. Śćiban; Stara Planina: Oreovica 15.07.2013. leg. M. Šćiban; Arilje: Roge 20.05.2018. leg. B. Nadaždin; Surdulica: Masurica 25.04.2019. leg. B. Nadaždin; Vlasina: Veliki Čemernik 25.05.2019. legs. I. Tot, M. Vujić, T. Tot, A. Đukić; Vlasina: Dejanova Reka, r. 26.05.2019. leg. M. Vujić, 24.07.2019. leg. B. Nadaždin; Vlasina: Apartman Filipović 27.05.2019. leg. M. Vujić; Vlasina: Vlasina Rid 23.05.2019. leg. I. Tot, 20.07.2019. leg. A. Đukić; Vlasina: Polom 06.09.2019. leg. B. Nadaždin; Vlasina: Gadžini 03.09.2019. leg. M. Vujić; Pčinja: Šaprance 24.04.2019. leg. B. Nadaždin; Tutin: Draga 02.07.2019. leg. B. Nadaždin.

Ref.: Divac 1907, Horváth 1903, Kormilev 1936, Protić 2011a, Šeat 2011, 2013, 2017, Šeat, Nadaždin 2015, 2018.

57. Gerris (Gerris) gibbifer Schummel, 1832

NHMBEO: Delliblatski Pesak: Devojački Bunar 24.04.2006. 2ex. leg. A. Stojanović; Mt. Jastrebac: Jezero (lake) 18.04. 2007. 1ex., 07.05. 2007. 3ex., 13.05.2007. 2ex., 21.08.2007. 1ex., 17.09.2007. 1ex., 23.09.2007. 3ex., 14.10.2007. 3ex. leg. I. Parezanović; Lazarevac: Stubički Vis 12.06.2010. 1ex. leg. A. Stojanović.

Alciphron database - Srbobran 08.06.2013. leg. M. Šćiban; Vlasina: Veliki Čemernik 25.05.2019. legs. I. Tot, M. Vujić, T. Tot, A. Đukić; Sopotnica 17.08.2019. leg. B. Nadaždin.

Ref.: Horváth 1903, Kormilev 1936, Živojinović 1950, Protić 1985, 1990, 1992, 1998, Protić \& Živić 2007, Šeat2013, Šeat, Nadaždin 2015.

58. Gerris (Gerris) lacustris (Linnaeus, 1758)

NHMBEO: Beograd: Topčider 28.07.1987. 1ex. leg. A. Stojanović; Mala Moštanica: Žuti Brdo 13.05.2006. 1ex. leg. A. Stojanović. 
Alciphron database - Beograd: Banjička Šuma 17.02.2014. leg. M. Đurić (F); Brusnica 11.04.2015. leg. M. Miljević (F); Novi Sad: Ledinci 06.04.2008. leg. M. Janković (F); Sopotnica 15.07.2015. leg. J. Šeat; Stara Planina: Temska 15.07.2013., 22.07.2013. leg. M. Šćiban; Divčibare 05.05.2013. leg. G. Mesaroš; Vršac: Straža 09.06.2017. leg. Z. Gavrilović (F); Reljan 23.04.2019. leg. B. Nadaždin; Vlasina: Dejanova Reka, r. 26.05.2019. leg. M. Vujić; Vlasina: Suhi Dol 04.09.2019. leg. M. Vujić.

Ref.: Protić 1998, 2008, Šeat 2011, Šeat, Nadaždin 2015, Protić et al. 2017, 2017a.

59. Gerris (Gerris) odontogaster (Zetterstadt, 1828

NHMBEO: Kovin: Malo Bavanište 10.06.2007. 1ex. leg. Maja Obradović.

Alciphron database - Kanjiža 19.05.2012. leg. G. Mesaroš.

Ref.: Protić 1990, 1998, Protić \& Živić 2013.

60. Gerris (Gerris) thoracicus Schummel, 1832

NHMBEO: Beograd: Avala 25.06.1941. 15ex. leg. B. Petrov; Prokuplje 30.06.1941. 1ex. leg. B. Petrov; Beograd: Ada Ciganlija 06.1956. 4ex. leg. D. Čubrilović; Beograd: Topčider 28.07.1987. 2 ex. leg. A. Stojanović; Zlatar 28.07.1979. 3ex. leg. Lj. Tričković; Zlatar: Vilovo, Lošnica, r. 28.07.1979. 6 ex. Lj. Kašić; Tara: Kaluđerske Bare 02.08.1988. 2ex., 04.08.1988. 3ex. leg. Lj. Protić; Obrenovac: Konatice 22.07.1992. 1ex. leg. Lj. Protić; Beograd: Ritopek 20.06.1998. 1ex. leg. A. Stojanvić; Mala Moštanica: Žuti Brdo 29.04.2000. 1ex., 22.06.2002. 2ex., 13.05.2006. 2ex., 14.04.2007. 4 ex., 10.07.2010. 1ex. leg. A. Stojanović; Valjevo: Debelo Brdo 13.04.2002. 1ex., 14.04.2002. 3ex. leg. M. Vujanić; Progar: Bojčinska Šuma 22.03.2003. 1ex. leg. M. Vujanić; Avala: Trešnja 03.06.2003. 4ex. leg. Lj. Protić; Beljanica: Lisine 19.07.2004. 3ex. leg. M. Vujanić; Beljanica, Beljanička Reka, r. 21.07.2004. 4ex. leg. M. Vujanić; Miroč 19.07.2005. 2ex. leg. M. Vujanić; Deliblatski Pesak: Devojački Bunar 24.04.2006. 3ex. leg. A. Stojanović; Beograd: Veliko Selo 08.07.2006. 3ex. leg. A. Stojanović; Mt. Jastrebac: Jezero (lake) 13.05.2007. 1ex., 08.07.2007. 1ex. leg. I. Parezanović; Goč: Dobre Vode 10.05.2011. 2ex. leg. Č. Marković; Ljig: Kadina Luka 15.06.2013. 2ex. leg. A. Stojanović; Grocka: Vrčin 30.03.2014. 3ex., 07.04.2007. 5ex., 12.06.2014. 1ex., leg. A. Stojanović.

Alciphron database - Sopotnica 15.07.2015. leg. J. Šeat, 17.08.2019. leg. B. Nadaždin; Stara Planina: Rudinje 19.07.2013. leg. M. Šćiban; Pešter: Braćak 13.10.2018. leg. B. Nadaždin; Vlasina: Veliki Čemernik 
25.05.2019. legs. I. Tot, M. Vujić, T. Tot, A. Đukić; Tutin: Draga 02.07.2019. leg. B. Nadaždin.

Ref.: Divac 1907, Horváth 1903, 1918, Kormilev 1936, Živojinović 1950, Protić 1998, 2008, Protić \& Živić 2007, Šeat, Nadaždin 2015, Protić et al. 2017a.

61. Gerris (Gerriselloides) asper (Fieber, 1860)

Ref.: Protić 1990, Protić \& Živić 2013.

62. Gerris (Gerriselloides) lateralis Schummel, 1832

Alciphron database - Stara Pazova 20.03.2014. leg. B. Hric (F).

Ref.: Csiki 1940, Protić 1998, 2008, Protić et al. 2017a.

\section{DISCUSSION AND CONCLUSIONS}

Studies of aquatic and semiaquatic true bugs in Serbia started in the mid- $19^{\text {th }}$ century. The first data were included in papers by foreign entomologists (Fieber 1861, Horvath 1897), and later also in papers by own local experts (Divac 1907, Kormilev 1936, 1938, 1939, 1939a, 1943).

In recent years, online databases and photo-based data collection applications have been increasingly developed. Such tools turned out to be a very good method for collecting the basic data on species distribution, but we need to be very careful about photo-based identification and it should not replace the classic methods of identification through identification keys. This paper also includes a list of localities where true bugs were photographed, as an integral part of Alciphron database.

Summary of all analyzed data shows a list of 62 species recorded in Serbia, with 12 families: Nepidae (2), Belostomatidae (1), Corixidae (25), Naucoridae (1), Aphelocheiridae (1), Notonectidae (6), Pleidae (1), Mesoveliidae (1), Hebridae (3), Hydrometridae (2), Velidae (9) and Gerridae (10).

Two new species were recorded for the fauna of Serbia: Hesperocorixa moesta (Fieber, 1848) and Velia (Plesiovelia) mancinii mancinii Tamanini, 1947.

In the old material in the Entomological Collection of the Natural History Museum, specimens of Velia sp. were mostly labeled as Velia (Velia) rivulorum (Fabricius, 1775). The review including checking and redetermining each specimen has shown presence of three species where none was actually $V$. rivulorum, and these were: Velia (Plesiovelia) affinis filippii Tamanini, Velia (Plesiovelia) mancinii mancinii Tamanini and Velia (Plesiovelia) sauli Tamanini. 
For $V$. rivulorum, which is listed in the list of species, we only had data from very old papers (Horváth 1897, Csiki 1940, Kormilev 1936). According to Berchi \& Kment (2015), the record for V. rivulorum from Bazijaš (Horváth 1897) pertains to $V$. mancinii mancinii. At the time it was the first record for Serbia, but today Bazijaš is within the borders of Romania. Therefore the official first records for Serbia are from Prokuplje and Avala - the mountain near Belgrade.

The original range of $V$. mancinii is the Mediterranean and the southern part of European Russia (Tamanini 1951, Josifov 1986, Aukema \& Rieger 1995, Kment \& Beran 2011, Berchi \& Kment 2015). Records from Balkan Peninsula are generally quite old: Albania (Josifov 1986, Aukema \& Rieger 1995); Bulgaria - Kresna Gorge June $24^{\text {th }} 1938$ (Tamanini 1949, Josifov 1963, 1999, Kment \& Kolínova 2013); Croatia (Dalmatia) - Strobeč near Split April 12 1937 (Novak \& Wagner 1951); Republic of North Macedonia - Treska river in the north part October $26^{\text {th }} 1959$ (Wagner 1962); Serbia - Prokuplje, Beograd: Avala (this paper).

Hesperocorixa moesta was recorded at the site Dejanova Reka (river) at Vlasina (Fig. 4). This species mainly occurs in aquatic habitats that are temporary. Dejanova Reka is a narrow river that forms a humid peat habitat, and the whole area actually represents the remains of a former large peat bog. H. moesta was also recorded in the neighboring countries: Croatia, Bosnia and Herzegovina, North Macedonia and Slovakia (Protić 1998, Reduciendo Klementova et al. 2015).

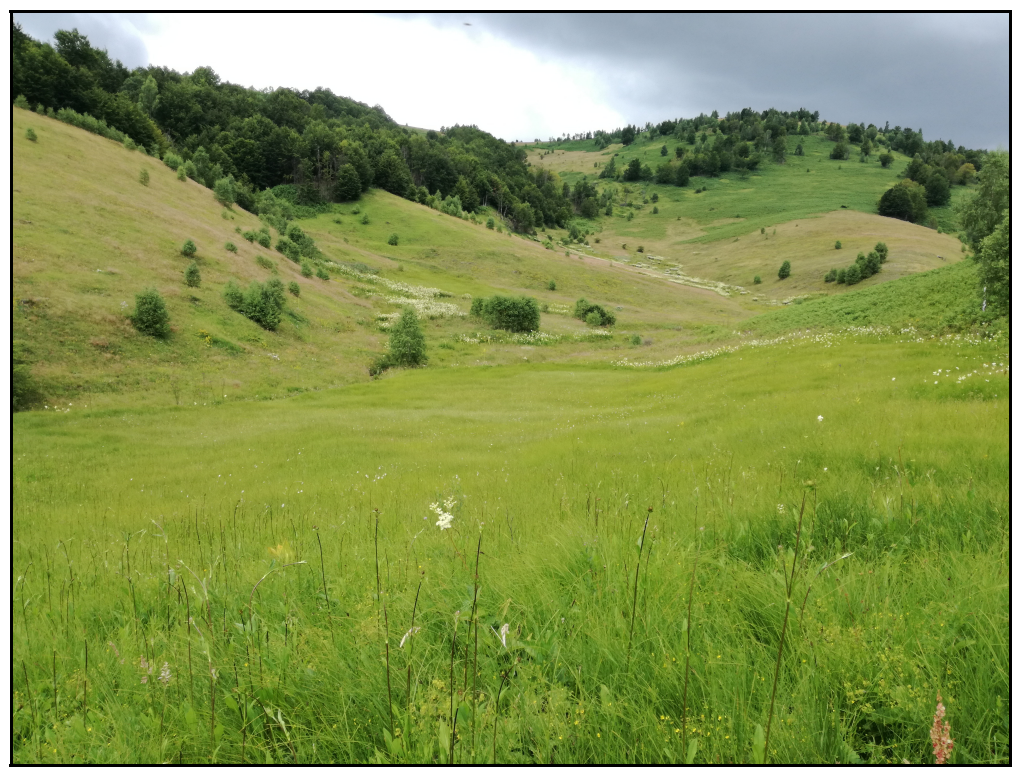

Fig. 4 - River Dejanova Reka (Vlasina) - Hesperocorixa moesta habitat (photo Bojana Nadaždin). 
The list of species includes one Balkan endemite - Velia serbica, as well as two glacial relicts - Hesperocorixa sahlbergi and Arctocorisa germari (Josifov \& Simov 2006, Beron 2016, Gueorguiev et al. 1998).

Lethocerus patruelis has so far been found in Serbia at only one site Obilić (Kormilev 1936). The record from Raška (locality Supnje) is only the second record after 79 years. This specimen is stored at the Entomological Collection of the Faculty of Science at the University of Kragujevac.

Some species were only mentioned in literature data, for example individual records of: Micronecta (Dichaetonecta) pusilla (Horváth) Horváth 1897; Micronecta (Dichaetonecta) scholtzi (Fieber) - Divac 1907; Sigara (Subsigara) distincta (Fieber) - Kormilev 1936; Microvelia (Microvelia) pygmaea (Dufour) - Horváth 1903.

There are 13 new species for the Catalogue of the Heteroptera of the Palearctic Region: Callicorixa praeusta praeusta (Fieber), Hesperocorixa moesta (Fieber), Hesperocorixa parallela (Fieber), Sigara (Retrocorixa) semistriata (Fieber), Sigara (Subsigara) iactans Jansson, Anisops sardeus Herrich-Schaeffer, Notonecta maculata Fabricius, Notonecta viridis Delcourt, Hydrometra gracilenta Horváth, Microvelia (Microvelia) pygmaea (Dufour), Velia (Plesiovelia) mancinii mancinii Tamanini, Velia (Plesiovelia) sauli Tamanini and Velia (Plesiovelia) serbica Tamanini.

Within the Table S1 occurred in Hungary, and the neighbouring countries Boda et al. (2015) listed three species for Serbia that are not included neither in our database nor in the Catalogue of the Heteroptera of the Palaearctic Region: Arctocorisa carinata carinata (C.R. Sahlberg), Sigara dorsalis (Leach) and Mesovelia vittigera Horváth.

We are witnessing the expansion of the range of individual Mediterranean species to a wider area of Europe, including Serbia (Rabitsch 2008, Protić 2011b, Šeat et al. 2019) and it is realistic to expect more of new species in national fauna in coming years. One good example is the expansion of Anisops sardeus Herrich-Schaeffer from the MeditteraneanCroatia, Island Šolta (Jaczewski 1934, Kment \& Beran 2011) toward the Central Europe. This species was recorded in: Bulgaria (Josifov 1955, Stoianova \& Simov 2014), southern Russia (Khatukhov et al. 2008), Hungary (Soos et al. 2010), Romania (Berchi 2011), northern Italy (Cianferoni \& Pinna 2012, Cianferoni \& Terzani 2013), Slovakia (Reduciendo \& Svitok 2014) and Serbia (Šeat et al. 2019).

The existing data are the result of individual samples and only a small number of watercourses in Serbia have been systematically studied. However, by analyzing the localities of existing records we concluded that 
most of Serbian territory has been covered and that diversity of aquatic habitats is high (Matvejev \& Puncer 1989, Lakušić et al. 2005).

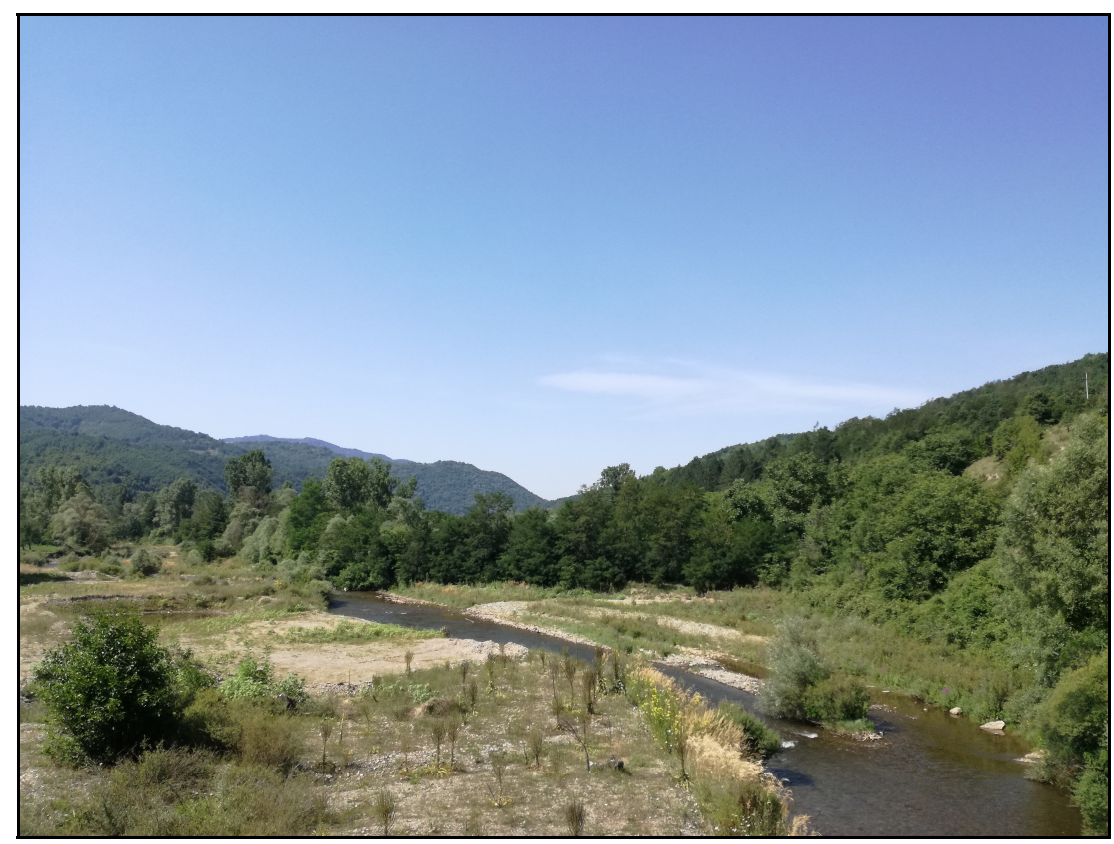

Fig. 5 - The Pčinja Valley (photo Bojana Nadaždin).

We believe that the number of 62 species is realistic for Serbia, considering the diversity of habitats from those at low altitudes (below 100 $\mathrm{m})$ to those above $1000 \mathrm{~m}$ a.s.l. This number should increase in the near future, as several young entomologists have joined the studies of heteropterans in Serbia in the last few years. Studies on some insufficiently explored areas (Valley of Pčinja River (Fig. 5), Vlasina and Pešter highland Plateau) started in the last two years and visits to new sites are planned. Therefore we expect records of new species and to complement the distribution of aquatic and semiaquatic true bugs in Serbia.

\section{REFERENCES}

Andersen, N. M. (1982): The Semiaquatic Bugs (Hemiptera, Gerromorpha): Phylogeny, Adaptations, Biogeography and Classification. - Entomonograph 3: $1-455$.

Andersen, N. M. (1996): Heteroptera Gerromorpha, Semiaquatic Bugs. In: Nilsson, A. N. (ed.): Aquatic Insects of North Europe - A Taxonomic Handbook. Apollo Books, Stenstrup. 
Aukema, B., Rieger, C. (eds) (1995): Catalogue of the Heteroptera of the Palaearctic Region 1. - The Netherlands Entomological Society, Amsterdam.

Aukema, B., Rieger, Ch., Rabitsch, W. (2013): Catalogue of the Heteroptera of the Palaearctic Region 6, Supplement. - The Netherlands Entomological Society, Amsterdam.

Benedek, P. (1970): The semiaquatic Heteroptera in the Carpathian Basin with notes on the distribution and the phenology of the species. - Faunistische Abhandlungen Staatliches Museum für Tierkunde in Dresden 3: 27-49.

Berchi, G. M. (2011): First record of Anisops sardeus (Hemiptera: Heteroptera: Notonectidae) in Romania. - North-Western Journal of Zoology 7: 339-341.

Berchi, G. M. (2013): Checklist and distribution of the family Notonectidae in Romania, with the first record of Notonecta maculata Fabricius, 1794 (Hemiptera: Heteroptera: Nepomorpha). - Zootaxa 3682(1): 121-132. [https:// doi.org/10.11646/zootaxa.3682.1.5]

Berchi, G. M., Kment, P. (2015): Review of the family Veliidae in Romania (Hemiptera: Heteroptera: Gerromorpha). - Zootaxa, 3963(1): 74-88. [https:// doi.org/10.11646/zootaxa.3963.1.5]

Berchi, G. M., Kment, P., Copilaş-Ciocianu, D., Rákosy, L., Damgaard, J. (2016): Water treaders of Romania and adjacent countries and their phylogenetic relationships (Hemiptera: Heteroptera: Mesoveliidae). - Annales Zoologici 66(2): 193-212.

Beron, P. (2016): Endemics and relicts in the high-mountain fauna of Bulgaria. Historia naturalis bulgarica 23: 109-118.

Boda, P., Bozóki, T., Vásárhelyi, T., Bakonyi, G., Várbíró, G. (2015): Revised and annotated checklist of aquatic and semi-aquatic Heteroptera of Hungary with comments on biodiversity patterns. - ZooKeys 501: 89-108. [https://doi.org/ 10.3897/zookeys.501.8964]

Cianferoni, F. (2013): Distribution of Cymatia rogenhoferi (Fieber, 1864) (Hemiptera, Heteroptera, Corixidae) in the West-Palaearctic Region, with the first record for the Italian mainland. - North-Western Journal ofZoology: 9(2): 245-249.

Cianferoni, F. (2019): Review of the aquatic Hemiptera from Cephalonia, with a checklist for the Ionian Islands, Greece (Heteroptera: Gerromorpha, Nepomorpha, Leptopodomorpha). - Zootaxa 4576(1): 46-60.

Cianferoni F., Nardi, G. (2013): Lethocerus patruelis (Stål, 1855) in Italy: a recent introduction or a natural westward spread? (Hemiptera: Heteroptera: Nepomorpha: Belostomatidae). - Zootaxa 3664: 78-84.

Cianferoni, F., Pinna, A. (2012): Segnalazioni Faunistiche Italiane. - Bollettino della Società entomologica italiana 144: 44-48.

Cianferoni, F., Terzani, F. (2013): Nuovi dati su Gerromorpha e Nepomorpha in Italia (Hemiptera: Heteroptera). - Bollettino della Società entomologica italiana 145(2): 51-57.

Csabai, Z., Soós, N., Berchi, G.M., Cianferoni, F., Boda, P., Mora, A. (2017): Aquatic and semiaquatic Heteroptera (Nepomorpha and Gerromorpha) fauna 
of Greek holiday islands (Rhodes, Crete and Corfu) with first records of three species from Europe and Greece. - Zootaxa, 4231 (1), 51-69.

Csiki, E. (1940): Félszárnyúrovarok. Hemipteren. Csiki Ernöállatanikutatásai Albániabán. Explorationes zoologicaeab E. Csiki in Albaniaperactae 17. - A Magyar Tudománios Akademia Balkán-kutatásainaktudománios Eredmenyei Budapest 1: 289-315.

Dahl, C. (2005): Heteroptera: Gerromorpha; Nepomorpha. - In: Nilsson, A. (ed.): The Aquatic Insects of North Europe 2: 77-103. - Apollo Books, Stenstrup.

Damgaard, J. (2006): Phylogeny and mtDNA phylogeography of two widespread European pond skater species (Hemiptera-Heteroptera: Gerridae: Gerris Fabricius). - Insect Systematics and Evolution 37: 335-350.

Divac, N. (1907): Prilog poznavanju srpske hemipterske faune. - Radovi iz zoološkog instituta u Univerzitetu Beograd 1(1): 4-14.

Fent, M., Kment, P., Çamur-Elípek, B., Kirgiz, T. (2011): Annotated catalogue of Enicocephalomorpha, Dipsocoromorpha, Nepomorpha, Gerromorpha, and Leptopodomorpha (Hemiptera: Heteroptera) of Turkey, with new records. Zootaxa 2856: 1-84.

Fieber, F. X. (1861): Die europäischen Hemipteren. - Druck und Verlag von Carl Gerold's Sohn, Wien.

Gueorguiev, V., Beshovski, V. L., Russev, B. K., Kumanski, K. P., Josifov, M. V., Sakalian, V. P. (1998): In: Sakahan, M. (ed.): Insects of Bulgaria, Part 1. Odonata, Ephemeroptera, Plecoptera, Homoptera (Auchenorrhyncha), Heteroptera, Coleoptera: 163-209. - Biodiversity Support Program. Conserving Biological Diversity in Bulgaria: The National Biological Diversity Conservation Strategy. Washington, D.C.: Biodiversity Support Program c/o World Wildlife Fund.

Gogala, A. (2003): Heteroptera of Slovenia I.: Dipsocoromorpha, Nepomorpha, Gerromorpha and Leptopodomorpha. - Annals for Istrian and Mediterranean Studies, Seria Historia Naturalis 13: 229-240.

HabiProt (2014-2019) Alciphron - baza podataka o insektima Srbije [http:// www.habiprot.org.rs/Alciphron] [accessed in November 2019]

Horváth, G. (1897): Fauna regni Hungariae. - In: Animalium Hungariaehucusque cogitorum enumeratio systematica 111. Arthropoda Ordo: Hemiptera: 1-72. Budapest.

Horváth, G. (1903): Szerbia Hemipter - faunaja. - Annales Musei Nationalis Hungarici 1: 3-28.

Horváth, G. (1918): Adatok a Balkán - Félsziget Hemiptera - Faunaja-nak Ismerétéhez. - Annales Musei Nationalis Hungarici 16: 321-340.

Jaczewski, T. (1934): Notes on some Palaearctic aquatic and semi-aquatic Heteroptera, chiefly from South-Eastern Europe. - Annales Musei Zoologici Polonici 10(14): 267-288.

Jaczewski, T. (1962): Gatunki rodzaju Velia Latr. Występujące w Polsce (Heteroptera, Veliidae). - Polskie Pismo Entomologiczne 32(18): 235-242. 
Jaczewski, T., Wróblewski, A. (1976): Hebridae, Mesoveliidae, Hydrometridae, Veliidae i nartniki - Gerridae. Klucze do oznaczania owadów polski. Tom. 18, Vol. 4. - Państwowe Wydawnictwo Naukowe, Warszawa, Wrocław.

Jaczewski, T., Wróblewski, A. (1978): Corixidae, Notonectidae, Pleidae, Nepidae, Naucoridae i Aphelocheiridae. Klucze do oznaczania owadów polski. Tom. 18, Vol. 2. - Państwowe Wydawnictwo Naukowe, Warszawa, Poznań.

Jansson, A. (1986): The Corixidae (Heteroptera) of Europe and some adjacent regions. - Acta Entomologica Fennica 47: 1-94.

Jansson, A. (1996): Heteroptera Nepomorpha, Aquatic Bugs. In: Nilsson, A. N. (ed.): Aquatic Insects of North Europe. A Taxonomic Handbook: 91-103. Apollo Books, Stenstrup.

Josifov, M. (1955): Hemiprologische mitteilung. - Bulletin de l'Institut de Zoologie et Musée 4(5): 461-462.

Josifov, M. (1963): Heteropteren aus der Umgebung von Petrie (SW Bulgarien). Izvestiya na Zoologicheskiya Institut, Sofia, 13: 93-132.

Josifov, M. (1986): Verzeichnis der von der Balkanhalbinsel bekannten Heteropterenarten (Insecta, Heteroptera). - Faunistische Abhandlungen Staatliches Museum für Tierkunde Dresden 14: 61-93.

Josifov, M. (1999): Heteropterous insects in the Sandanski-Petrich Kettle, Southwestern Bulgaria. - Historia Naturalis Bulgarica 10: 35-66.

Josifov, M., Simov, N. (2006): Endemism among the Heteroptera on the Balkan Peninsula. - Denisia 19: 879-898.

Kanyukova, E. V. (2006): Vodnye poluzhestkokrylye nasekomye (Heteroptera: Nepomorpha, Gerromorpha) fauny Rossii i sopredelnykh stran. - Dal'nauka, Vladivostok.

Khatukhov A. M., Yakimov A. V., Lvov V. D. (2008): Backswimmers (Heteroptera: Notonectidae) of the Central Caucasus (territory of the KabardinoBalkarian Republic). - Bulletin of the Adyghe State University Maikop 4: 117-119.

Kment, P. (2006): On the distribution of Micronecta carpatica Wróblewski, 1958 (Heteroptera: Corixidae). - Acta entomologica serbica 11: 101-104

Kment, P., Beran, L. (2011): Check-list of water bugs (Hemiptera: Heteroptera: Nepomorpha) in Croatia with two new records and four rediscoveries. - Natura Croatica 20(1): 159-178.

Kment, P., Bryja, J, Jindra, Z. (2005): New records of true bugs (Heteroptera) of the Balkan Peninsula. - Acta entomologica slovenica 13(1): 9-20.

Kment, P., Jindra, Z. (2005): New and interesting records of true bugs (Heteroptera) from Turkey, southeastern Europe, Near and Middle East. - Acta Entomologica Musei Nationalis Pragae 45: 3-16.

Kment, P., Kolínova, Z. (2013): Catalogue of type specimens of true bugs (Hemiptera: Heteroptera) deposited in the National Museum, Prague, Czech Republic. Enicocephalomorpha, Dipsocoromorpha, Nepomorpha, Gerromorpha, and Leptopodomorpha. - Acta Entomologica Musei Nationalis Pragae 53: 821-890. 
Kormilev, N. (1936): 1. prilog poznavanju Hemiptera- Heteroptera Jugoslavije (Južna Srbija i Srbija). - Glasnik skopskog naučnog društva 17(5): 29-54.

Kormilev, N. (1938): II. Beitrag zur Kenntnis der Verbbreitung jugoslavisher Hemiptera- Heteroptera (Serbien und Südserbien). - Glasnik skopskog naučnog društva 18(6): 167-172.

Kormilev, N. (1939): III. Beitrag zur Kenntnis der Verbreitung jugoslavischer Hemiptera - Heteroptera (Serbien und Südserbien). - Annales musei Serbie Meridionalis 1(2): 21-29.

Kormilev, N. (1939a): IV. Beitrag zur Kenntnis der Verbreitung jugoslavischer Hemiptera - Heteroptera (Serbien und Südserbien). - Glasnik skopskog naučnog društva 20(7): 193-199.

Kormilev, N. (1943): V. Beitrag zur Kenntnis der Verbreitung balkanischer Hemiptera - Heteroptera (Serbien und Mazedonien). - Ohridski zbornik 35, Srpska kraljevska Akademija, posebna izdanja 86, Prirodnjački i matematički spisi 6: 123-132.

Lakušić, D., Blaženčić, J, Ranđelović, V., Butorac, B., Vukojičić, S., Zlatković, B., Jovanović, S., Šinžar-Sekulić, J, Žukovec, D., Ćalić, I, Pavićević, D. (2005): Staništa Srbije. Priručnik sa opisima i osnovnim podacima. - Rezultati projekta „Harmonizacija nacionalne nomenklature u klasifikaciji staništa sa standardima međunarodne zajednice“. Institut za Botaniku i Botanička Bašta „Jevremovac“, Biološki fakultet, Univerzitet u Beogradu, Ministarstvo za nauku i zaštitu životne sredine Republike Srbije. [http://www.ekoserb.sr. gov.yu/projekti/stanista/, http://habitat.bio.bg.ac.yu/]

Langhoffer, A. (1899): Prilozi entomološkoj fauni Hrvatske. - Rad Jugoslavenske akademije znanosti i umjetnosti 141: 11-26.

Matvejev, S. D., Puncer, I. J. (1989): Map of Biomes. Landscapes of Yugoslavia and their protection. - Natural History Museum, Belgrade. Special issue 36: 1-76.

Novak, P., Wagner, E. (1951): Prilog poznavanju faune Hemiptera Dalmacije (Hemiptera - Heteroptera). - Godišnjak Biološkog instituta u Sarajevu 4(1): 59-80.

Poisson, R. (1957): Hétéroptères aquatiques. Faune de France 61. - Fédération Française des Sociétés de Sciences Naturelles, Paris.

Protić, Lj. (1985): Zbirka Heteroptera Hilfa Morica iz Srbije u Zemaljskom muzeju Bosne i Hercegovine u Sarajevu. - Glasnik Zemaljskog muzeja Bosne I Hercegovine, Prirodne nauke, Nova serija 24: 153-161.

Protić, Lj. (1986): Prvi prilog poznavanju faune Hemiptera - Heteroptera Deliblatskog peska. - Deliblatski pesak, Zbornik radova 5: 203-232.

Protić, Lj. (1990): Zbirka Hemiptera - Heteroptera Nikole A. Kormileva u Prirodnjačkom muzeju u Beogradu. Drugi deo. - Habilitacioni rad za zvanje viši kustos. (manusc.)

Protić, Lj. (1992): Materials for the Heteroptera fauna of North - Eastern Serbia. Bulletin of the Natural History Museum in Belgrade B 47: 193-245.

Protić, Lj. (1993/1994): Check List of the Heteroptera Fauna of Yugoslav countries. - Bulletin of the Natural History Museum in Belgrade B 48: 33-61. 
Protić, Lj. (1998): Catalogue of the Heteroptera fauna of Yugoslav countries. Part one. - Natural History Museum, Belgrade. Special issue 38: 1-215.

Protić, Lj. (2008): Old collection of Heteroptera at the Natural History Museum in Belgrade. - Bulletin of the Natural History Museum in Belgrade 1: 187-203

Protić, Lj. (2011a): Heterptera. Prirodnjački muzej, Posebna izdanja, 43: 1-259.

Protić, Lj. (2011b): New Heteroptera for the fauna of Serbia. - Bulletin of the Natural History Museum in Belgrade, 4: 119-125.

Protić, Lj. (2019): Istraženost stenica (Heteroptera) vodenih staništa Srbije. In: Žikić, V. (ed.): XII Simpozijum entomologa Srbije sa međunarodnim učešćem. Zbornik rezimea Niš, 25-29.IX 2019: 12. - Entomološko društvo Srbije, Beograd.

Protić, Lj., Živić, I. (2007): New data on water bugs (Heteroptera) in Serbia. - Acta entomologica serbica 12(2): 17-26.

Protić, Lj., Živić, N. (2012): Water bugs (Heteroptera) in the catchment area of river Sitnica (Serbia). - Acta entomologica serbica 17 (1-2): 29-37.

Protić, Lj., Živić, N. (2013): A contribution to the knowledge of aquatic Heteroptera in Šar Planina Mts. and Pešter Plateau (Serbia). - Acta entomologica slovenica 21(1): 65-76.

Protić, Lj., Savić, D., Stojanović, A. (2017): Heteroptera of the Fruška Gora Mountain. - In: Šimić, S. (ed.): Invertebrates (Invertebrata) of the Fruška Gora Mountain 5: 47-104. - Matica srpska, Novi Sad.

Protić, Lj., Šćiban, M., Šeat, J., Stanković, M. (2017a): Fauna stenica (Insecta: Heteroptera) Specijalnog rezervata prirode „Zasavica“. In: Simić, S. (ed.): Naučno-stručni skup o biodiverzitetu i drugim vrednostima rezervata Zasavica, „Zasavica 2017“ sa međunarodnim učešćem: 117-127. - Pokret gorana Sremska Mitrovica, Sremska Mitrovica.

Rabitsch, W. (2005): Bestimmungsschlüssel der Nepomorpha Österreichs. Spezialpraktikum aquatische und semiaquatische Heteroptera SS 2005: 18-46. [http://homepage.univie.ac.at/ wolfgang.rabitsch/Bestimmungsschluessel_comb.pdf]

Rabitsch, W. (2008): The times they are a-changin': driving forces of recent additions to the Heteroptera fauna of Austria. In: Grozeva S., Simov N. (eds): Advances in Heteroptera Research, Festschrift in Honour of $80^{\text {th }}$ Anniversary of Michail Josifov: 309-326. - Pensoft, Sofia, Moscow.

Rabitsch, W., Zettel, H. (2000): Zur Wasserwanzenfauna (Heteroptera: Gerromorpha und Nepomorpha) des nördlichen Österreich. - Linzer Biologische Beiträge 32: 1257-1268.

Reduciendo Klementová, B., Svitok, M. (2014): Anisops sardeus (Heteroptera): A new expansive species in Central Europe. - Biologia (Bratislava) 69: 676-680. [http://dx.doi.org/10.2478/s11756-014-0354-z]

Reduciendo Klementova, B., Kment, P., Svitok, M. (2015): Checklist of water bugs (Hemiptera: Heteroptera: Nepomorpha, Gerromorpha) of Slovakia. Zootaxa 4058(2): 227-243.

Savage, A. A. (1989): Adults of the British aquatic Hemiptera Heteroptera. A key with ecological notes. - Freshwater Biological Association, Ambleside. 
Savage, A. A. (1990): A key to the adults of British Lesser water boatmen (Corixidae). - Field Studies 7: 485-515.

Schumacher, F. (1918): Beiträge zur Kenntnis der Hemipterenfauna Mazedoniens. - Sitzungsberichte der Gesellschaft Naturforschender Freunde zu Berlin 1918: 82-98.

Schuster G. (1983): Zur Trennung von Gerris lateralis Schumm. und Gerris asper Fieb. - Berichten der Naturforschenden Gesellschaft Augsburg 38: 3-6.

Soós, N., Boda, P., Csabai, Z. (2009): First confirmed occurrences of Notonecta maculata and N. meridionalis (Heteroptera: Notonectidae) in Hungary with notes, maps, and a key to the Notonecta species of Hungary. - Folia Entomologica Hungarica 70: 67-78.

Soós, N., Petri, A., Nagy-László, Zs., Csabai, Z. (2010): Anisops sardeus HerrichSchaeffer, 1849: first records from Hungary (Heteroptera: Notonectidae). Folia Entomologica Hungarica 71: 15-18.

Stichel, W. (1955-1956): Illustrierte Bestimmungstabellen der Wanzen. II. Europa (Hemiptera- Heteroptera Europae) 1. - Berlin, Hermsdorf.

Stoianova, D., Simov, N. (2014): New records of Aquatic True Bugs (Hemiptera: Heteroptera: Nepomorpha) from Bulgaria. - Acta Zoologica Bulgarica 68(4): 497-502.

Šeat, J. (2011): True bugs (Heteroptera) of Pčinja Valley (Serbia). - Acta entomologica serbica 16(1-2): 9-24.

Šeat, J. (2013): True bugs (Heteroptera) of Stara Planina Mountain (Serbia). - Acta entomologica serbica 18 (1-2): 17-41.

Šeat, J. (2017): Contribution to the knowledge on true bugs (Insecta: Heteroptera) of Vlasina. - Zaštita prirode 67 (1-2): 43-54.

Šeat, J., Nadaždin, B. (2015): Overview of the true bug (Heteroptera) fauna of the Ovčar-Kablar Gorge. - Beležnik Ovčarsko-kablarske klisure 6 (1): 38-47.

Šeat, J., Nadaždin, B., Cvetković, M., Jovanov, A., Tot, I. (2016): Providing a base for conservation of true bugs (Insecta, Heteroptera) and their saline habitats in Vojvodina (northern Serbia). - Hyla 2016(1): 19-23.

Šeat, J., Nadaždin, B. (2018): The true bugs (Insecta, Heteroptera) of the OvčarKablar Gorge. - Beležnik Ovčarsko-kablarske klisure 8: 8-16.

Šeat, J., Vujić, M., Nadaždin, B. (2019): New faunal data on true bugs (Heteroptera). - Acta entomologica serbica 24 (1): 95-99.

Tamanini, L. (1949) Secondo contributo allo studio del genere Velia Latr. (Heteroptera: Hydrometridae). - Bollettino della Società Entomologica Italiana 79: $35-40$.

Tamanini, L. (1951) [1949]: Terzo contributo allo studio del genere Velia Latr. (Hemipt.-Heteropt., Veliidae). - Acta Entomologica Musei Nationalis Pragae 26(366) : 1-10.

Tamanini, L. (1959) [1957]: Valore tassonomico della Velia serbica Tam. e brevi osservazioni sulle Velia della Bulgaria. - Atti della Accademia Roveretana degli Agiati, Series 5, 6: 131-135. 
Wachmann, E., Melber, A., Deckert, J. (2006): Die Tierwelt Deutschlands 77. Teil. Wanzen Band 1. Dipsocoromorpha, Nepomorpha, Gerromorpha, Leptopodomorpha, Cimicomorpha (Teil 1). - Keltern, Goecke, Evers.

Wagner, E. (1962): 2. Beitrag zur Heteropteren - fauna Macedoniens (Hem. Het.). - Fragmenta Balcanica. Musei Macedonici Scientiarum naturalium Skopje 4, 15 (99): 115-122.

Wróblewski, A. (1960): Micronectinae (Heteroptera, Corixidae) of Hungary and of some adjacent countries. - Acta Zoologica Academiae Scientiarum Hungaricae 6: 441-458.

Wróblewski, A. (1980): Pluskwiaki (Heteroptera). Fauna słodkowodna Polski 8. Państwowe Wydawnictwo Naukowe, Warszawa, Poznań.

Živić, I., Protić, Lj., Marković, Z. (2007): Southernmost finding in Europe of Aphelocheirus aestivalis (Fabricius, 1794) (Hemiptera: Heteroptera: Aphelocheiridae). - Zootaxa 1496: 63-68.

Živojinović, S. (1950): Fauna insekata šumske Domene Majdanpek. Entomološka monografija. - Srpska Akademija nauke i umetnosti, Institut za ekologiju i biogeografiju. Posebna izdanja 160(2): 1- 262.

Zimmermann, G. (1982): Beiträge zur Wasserwanzen-Fauna Griechenlands I (Heteroptera, Nepomorpha, Gerromorpha, Saldidae). - Marburger Entomologische Publikationen 1(6): 183-216.

Zimmermann, M., Scholl, A. (1993): Specific status of Aquarius cinereus (Puton) and najas (De Geer) in the Mediterranean region. - Entomologica Scandinavica 24: 197-210.

\title{
ВОДЕНЕ СТЕНИЦЕ У СРБИЈИ
}

ЉИљАНА ПРОТИЋ И БОЈАНА НАДАЖДИН

\author{
Р Е 3 И М Е
}

Истраживања стеница (Heteroptera) водених, влажних и мочварних станишта су годинама у Србији била запостављена у односу на терестрична станишта. Иако први подаци о воденим стеницама потичу из половине 19. века, систематских истраживања није било. Последњих година све је више истраживача у Србији који обраћају пажњу и на истраживање и сакупљање водених стеница (Nepomorpha и Gerromorpha). 
Циљ рада је дати приказ врста водених стеница Србије на основу примерака из збирки Природњачког музеја у Београду, базе „Alciphron“ и литературе.

Састављен је списак који укључује 62 врсте. Пронађене су две нове врсте за фауну Србије: Hesperocorixa moesta (Fieber, 1848) и Velia (Plesiovelia) mancinii mancinii Tamanini, 1947. Од укупног броја наведених врста једна је балкански ендемит - Velia serbica Tamanini, 1951 а две су глацијални реликти - Hesperocorixa sahlbergi (Fieber, 1848) и Arctocorisa germari (Fieber, 1848). 